\title{
Pilot studies of age-related changes in blood perfusion in two different types of skin
}

Yulia I. Loktionova, Elena V. Zharkikh, Igor O. Kozlov, Evgeny A. Zherebtsov, Svetlana A. Bryanskaya, et al.

Yulia I. Loktionova, Elena V. Zharkikh, Igor O. Kozlov, Evgeny A. Zherebtsov, Svetlana A. Bryanskaya, Angelina I. Zherebtsova, Victor V. Sidorov, Sergei G. Sokolovski, Andrey V. Dunaev, Edik U. Rafailov, "Pilot studies of age-related changes in blood perfusion in two different types of skin," Proc. SPIE 11065, Saratov Fall Meeting 2018: Optical and Nano-Technologies for Biology and Medicine, 110650S (3 June 2019); doi: 10.1117/12.2522968 


\title{
Pilot studies of age-related changes in blood perfusion in two different types of skin
}

\author{
Yulia I. Loktionova ${ }^{\mathrm{a}}$, Elena V. Zharkikh ${ }^{\mathrm{a}}$, Igor O. Kozlov ${ }^{\mathrm{a}}$, Evgeny A. Zherebtsov ${ }^{\mathrm{a}, \mathrm{b}}$, \\ Svetlana A. Bryanskaya ${ }^{a}$, Angelina I. Zherebtsova ${ }^{a}$, Victor V. Sidorov ${ }^{c}$, Sergei G. Sokolovski ${ }^{\mathrm{d}}$, \\ Andrey V. Dunaev ${ }^{\mathrm{a}}$, and Edik U. Rafailov ${ }^{\mathrm{d}}$ \\ ${ }^{a}$ Orel State University named after I.S. Turgenev, 95 Komsomolskaya St, Orel, 302026, Russia \\ ${ }^{\mathrm{b} U n i v e r s i t y ~ o f ~ O u l u, ~ O u l u, ~ 90570, ~ F i n l a n d ~}$ \\ "SPE "LAZMA", Moscow, Russia \\ ${ }^{\mathrm{d}}$ Aston Institute of Photonic Technologies, Aston University, Aston Triangle, Birmingham, B4 \\ $7 \mathrm{ET}, \mathrm{UK}$
}

\begin{abstract}
Laser Doppler flowmetry (LDF) was utilized to assess age-related changes in the blood microcirculation at the skin sites with different morphology and regulation. The LDF signals obtained from the glabrous skin of the middle finger pad and nonglabrous skin on the dorsal wrist surface were analyzed. Statistically higher baseline perfusion was observed in the zone with glabrous skin in the older group of volunteers compared to younger participants. Observed site-specific and age-related differences in perfusion can be used in the future experimental design for the studies of the blood microcirculation system in patients with different pathologies.
\end{abstract}

Keywords: laser Doppler flowmetry, blood microcirculation, ageing

\section{INTRODUCTION}

With age, many body systems change structurally and functionally. The vascular bed is one of the first targets of age-related changes. ${ }^{1}$ Numerous studies of age-related changes in microcirculation have revealed significant deterioration of trophic support of tissues and organs, and consequently, a great influence of ageing on the pathogenesis of various diseases..$^{2-4}$ Therefore, studies of age-related changes in blood flow can be used to identify diseases and disorders in the functioning of the cardiovascular and other systems of the body.

In modern diagnostics, there are many different optical non-invasive methods allowing ones to measure blood perfusion. Analysis of time series records of the blood perfusion is a useful approach to evaluate the overall functional state of the vascular system. ${ }^{5}$ One of these methods is the method of laser Doppler flowmetry (LDF). The technology is based on optical non-invasive sensing of tissues by the NIR single mode laser, followed by the analysis of backscattered light reflected from moving red blood cells. The LDF method is used for functional diagnostics of the blood microcirculation system, including the diagnosis of socially significant diseases associated with the cardiovascular system, and complications arising during their course. Such diseases include diabetes ${ }^{6,7}$ psoriasis $^{8}$ and rheumatological diseases. ${ }^{9-11}$ Also, this method allows one to estimate the oscillatory processes in the microvessels. There are several frequency ranges characterizing the contribution of various factors to the LDF signal: endothelial $(0.0095-0.021 \mathrm{~Hz})$, neurogenic $(0.021-0.052 \mathrm{~Hz})$, myogenic $(0.052-0.145 \mathrm{~Hz})$, respiratory $(0.145-0.6 \mathrm{~Hz})$ and cardiac $(0.6-2 \mathrm{~Hz}) .{ }^{12}$ Fig. 1 shows a typical view of the LDF record and its amplitude-frequency spectrum.

By now, the LDF method has been applied in the studies of age-related changes in blood flow as well. The studies have been conducted both at rest and with various functional tests. ${ }^{13-15}$ Local heating and occlusion tests $^{16}$ are most commonly used among them. Usually in that tests, the maximum peak of blood flow is assessed, achieving by locally applied skin moderate heating or during post-occlusion hyperemia.

Send correspondence to Yulia Loktionova

E-mail: julya-loktionova@mail.ru

Saratov Fall Meeting 2018: Optical and Nano-Technologies for Biology and Medicine, edited by

Elina A. Genina, Valery V. Tuchin, Proc. of SPIE Vol. 11065, 110650S · C 2019 SPIE

CCC code: $0277-786 X / 19 / \$ 18 \cdot$ doi: $10.1117 / 12.2522968$

Proc. of SPIE Vol. 11065 110650S-1 

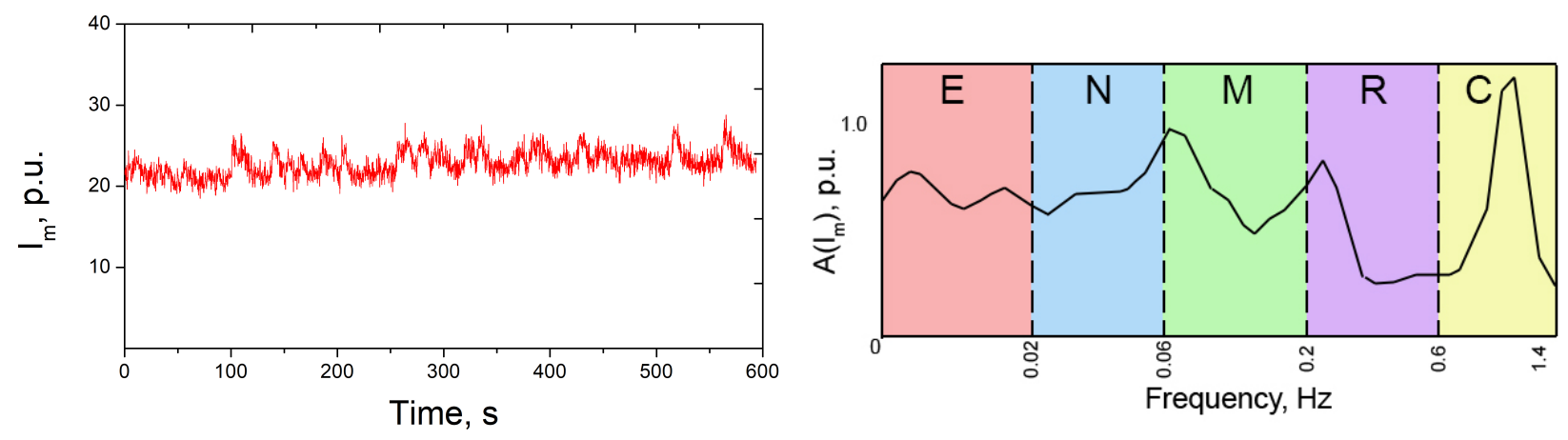

Figure 1. The typical view of LDF-record (left panel) and an amplitude-frequency spectrum of the obtained signal (right panel)

However, there is a shortage of studies devoted to the quantitative assessment of age-related microcirculatory changes in vivo in different anatomical sites.

This work aimed to study age-related changes of LDF signals simultaneously registered in the skin of limbs in healthy volunteers of different age groups.

\section{MATERIALS AND METHODS}

Experimental studies were carried out using four wearable laser Doppler flowmetry monitors "AMT-LAZMA 1" (Aston Medical Technology Ltd., UK) for blood microcirculation analysis. Devices implement identical channels for blood perfusion registration and allow simultaneous measurements in several points of the body. At the moment these devices are the latest development to assess the state of blood microcirculation. ${ }^{17}$

These studies were performed by the principles outlined in the 2013 Declaration of Helsinki by the World Medical Association. The studies were approved by the Ethical Committee of the Orel Regional Clinical Hospital. The study involved 36 healthy volunteers without cardiovascular diseases, hypertension, diabetes and other serious chronic diseases that affect the blood microcirculation system. Before starting the study, each volunteer gave voluntary informed written consent to participate in the experiment. Volunteers also filled out a questionnaire to identify possible health problems. Volunteers with a history of alcohol, medicamental, or drug abuse were also excluded from the study. The study participants were divided into two groups according to their age: 18 people were included in the group of volunteers younger than 20 years (1st group: the average age is $19.4 \pm 0.6$ years), 18 volunteers were over 40 years (2nd group: the average age is $52.6 \pm 10.2$ years). The physical characteristics of the two groups under the study are presented in Table 1. Apart from age, there was no significant difference in other parameters according to enrollment criteria.

Table 1. Participant characteristics

\begin{tabular}{|l|l|l|}
\hline Parameter & 1st group & 2nd group \\
\hline Age, years & $19.4 \pm 0.6^{*}$ & $52.6 \pm 10.2$ \\
\hline Pulse & $79.6 \pm 9.2$ & $74.3 \pm 9.1$ \\
\hline Systolic pressure & $121.1 \pm 7.8$ & $125.0 \pm 7.8$ \\
\hline Diastolic pressure & $77.4 \pm 5.3$ & $78.1 \pm 7.2$ \\
\hline
\end{tabular}

* - The significance of the difference between the values was confirmed with $p<0.05$ using Mann-Whitney test

Studies were conducted in the sitting position, in a state of physical and mental rest not earlier than 2 hours after eating. Volunteers hands were placed on the table at heart level. The blood microcirculation index was recorded for 10 minutes, while sensors were attached to the palmar surface of the distal phalanx of the 3rd fingers of hands and the dorsal surface of forearms without applying any pressure on the study area.

Fig. 2 shows the location of the devices during the study. 


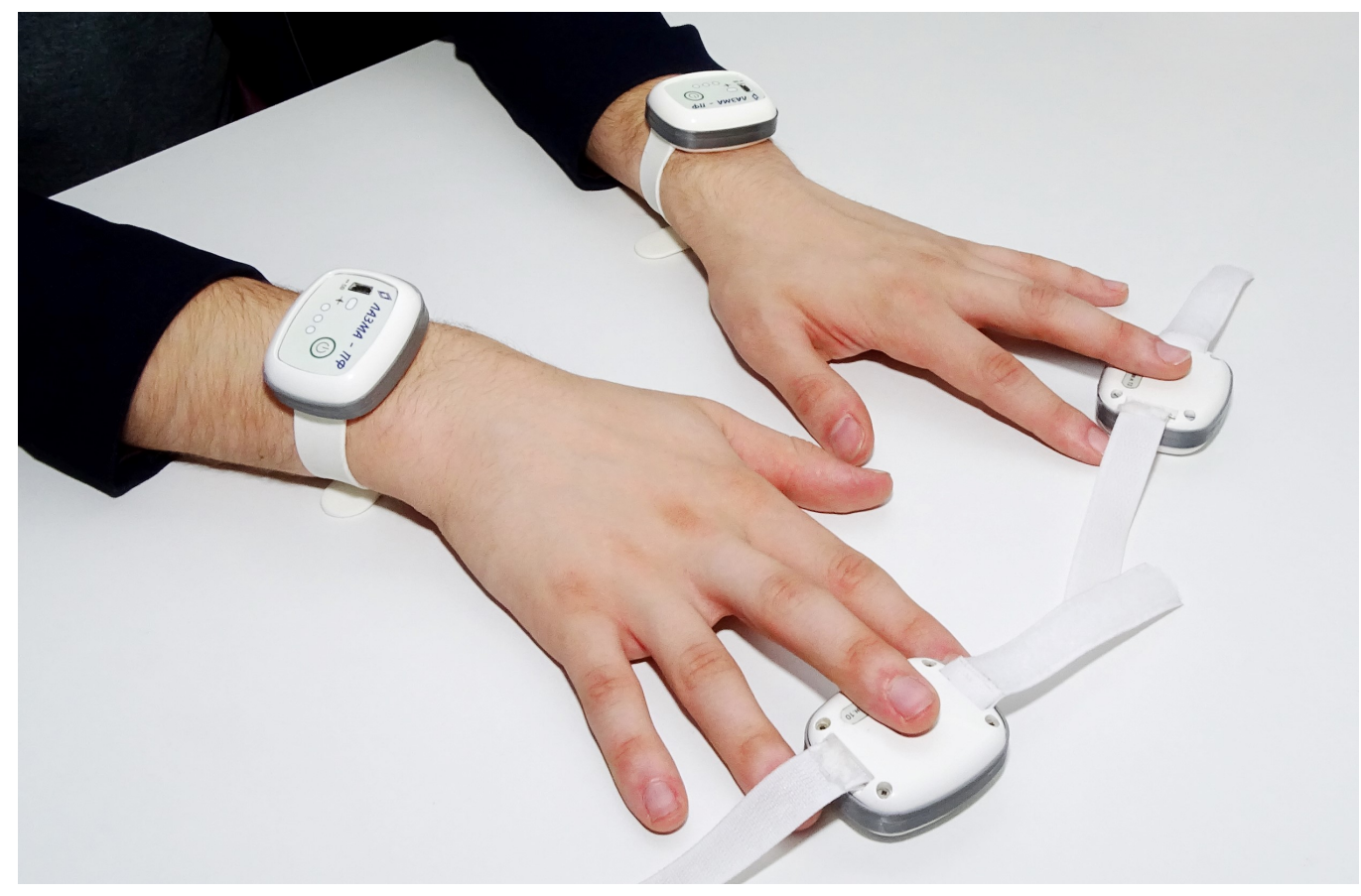

Figure 2. Location of the sensors

These areas were chosen because they represent two main skin types: glabrous and nonglabrous skin. Glabrous skin mainly covers the palms, soles and face. This skin type is primarily involved in body thermoregulation mechanisms. It contains a large number of arteriovenous anastomoses (AVAs) maintained in the constricted state by sympathetic tone. Thus, the sympathetic regulation is the dominant mechanism involved in the blood flow in the glabrous skin. ${ }^{18}$ Nonglabrous or "hairy" skin covers almost the entire surface of the human body. It contains only a few AVAs, so that blood flow there is primarily nutritive in function. The reflex nervous regulation of this skin type involves both sympathetic noradrenergic vasoconstrictor nerves and separate sympathetic cholinergic vasodilator nerves. ${ }^{19}$ The majority of studies devoted to age-related changes in microcirculatory blood flow was focused on the nonglabrous skin with less attention to glabrous skin sites.

\section{RESULTS AND DISCUSSION}

As mentioned earlier, the volunteer groups were recruited in such a way that the second group was significantly older than the first $(p<0.05)$. While all volunteers were normotensive, there was no significant difference in blood pressure and heart rate parameters.

Fig. 3 shows the results of measuring the average level of tissue perfusion with blood on the fingertip (a) and forearm (b) for two groups of subjects. The volunteers in the older group had a higher blood perfusion level in fingers in comparison to volunteers in the younger group (18.23 \pm 2.97 p.u. vs $14.26 \pm 4.68$ p.u.). There was observed a similar situation with the higher perfusion in older volunteers $(7.28 \pm 2.21$ p.u. vs $6.35 \pm 1.88$ p.u.) in the forearms, but this difference did not reach a statistically significant level.

Previous studies of age-related changes in microcirculation using non-invasive techniques have mainly focused on the application of various functional effects, like temperature, occlusion, etc. ${ }^{14,20}$ Only a few papers considered the difference in LDF signals of different age groups under basal conditions. ${ }^{13,21}$ Most studies evaluated the level of perfusion in areas with nonglabrous skin, where there was not detected any statistically significant difference in basal blood flow between younger and older participants. These data completely coincide with our microcirculation measurements on the dorsal side of the wrists obtained in this study.

In contrast, significantly higher perfusion values were observed in the glabrous skin of the older age group in comparison to younger participants when experiments were conducted on the third finger pad. The positive 

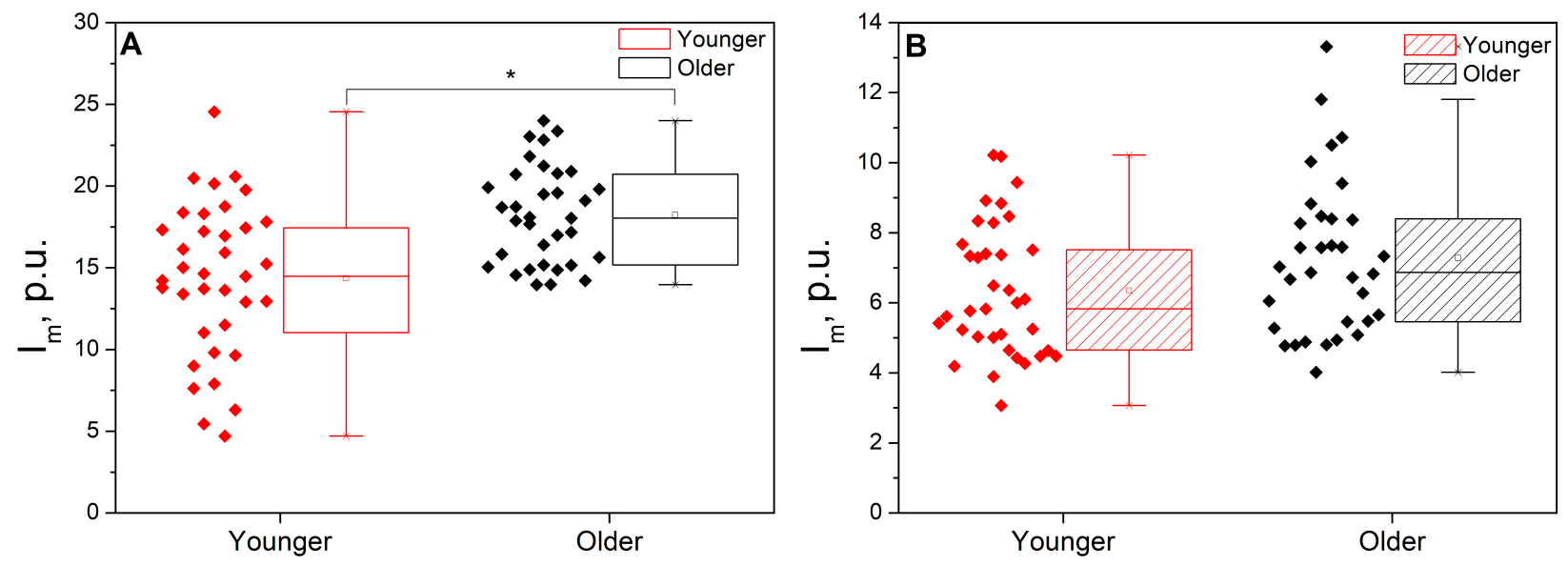

Figure 3. The microcirculation index values for the 1st (red boxes) and 2nd (black boxes) groups studied during measurements on the fingertip (figure a) and the dorsum of the forearm (figure b).

* - The significance of the difference between the values was confirmed with $p<0.05$ using Mann-Whitney test

correlation between age and basal blood flow levels measured by LDF is confirmed by previous studies. ${ }^{1,13}$ This result might be due to structural changes in microcirculation during ageing, including an increase in the total parallel vascular length. ${ }^{1}$

A higher signal level in the older group can also be explained by skin thinning during the ageing process, as a result of which laser radiation is less scattered, increasing the diagnostic volume. ${ }^{20}$ Whether the resulting data of higher perfusion in the older group is a result of structural and functional changes in microcirculation during ageing or the effect of a greater penetration depth of laser radiation into the skin, is the subject of further investigation.

\section{CONCLUSIONS}

The blood microcirculation system is subject to significant structural and functional changes during aging. Presented study has shown a higher level of microcirculation in areas with glabrous skin in the older age group. The measurements made on the forearms demonstrate the same tendency. However, the difference in the parameters of the groups does not reach a statistically significant level.

To clarify the results and their interpretation, it is necessary to continue research with an increase in the samples number and size. The use of wavelet analysis of the received LDF signals to detect changes in the microcirculation regulatory mechanisms during ageing is also promising. The obtained data can be considered further in the development of protocols for the studies of the blood microcirculation system in patients with different pathologies.

\section{ACKNOWLEDGMENTS}

This study was funded by the Russian Science Foundation (the research project 18-79-00237).

\section{REFERENCES}

[1] Li, L., Mac-Mary, S., Sainthillier, J.-M., Nouveau, S., Lacharriere, O. D., and Humbert, P., "Age-related changes of the cutaneous microcirculation in vivo," Gerontology 52(3), 142-153 (2006).

[2] Luscher, T. and Noll, G., "Endothelial function as an end-point in interventional trials: concepts, methods and current data," Journal of hypertension, Supplement 14(2), S111-S121 (1996).

[3] Tanaka, H., Tanaka, F., Monahan, K., Clevenger, C., Desouza, C., and Seals, D., "Aging, habitual exercise, and dynamic arterial compliance," Circulation (11), 1270-5 (2000). 
[4] Makrantonaki, E. and Zouboulis, C., "Characteristics and pathomechanisms of endogenously aged skin," Dermatology 214(4), 352-360 (2007).

[5] Daly, S. M. and Leahy, M. J., "Go with the flow": A review of methods and advancements in blood flow imaging," Journal of Biophotonics 6(3), 217-255 (2013).

[6] Mizeva, I., Zharkikh, E., Dremin, V., Zherebtsov, E., Makovik, I., Potapova, E., and Dunaev, A., "Spectral analysis of the blood flow in the foot microvascular bed during thermal testing in patients with diabetes mellitus," Microvascular Research 120, 13 - 20 (2018).

[7] Zharkikh, E., Dremin, V., Filina, M., Makovik, I., Potapova, E., Zherebtsov, E., Zherebtsova, A., and Dunaev, A., "Application of optical non-invasive methods to diagnose the state of the lower limb tissues in patients with diabetes mellitus," Journal of Physics: Conference Series 929(1), 012069 (2017).

[8] Dremin, V. V., Filina, M. A., Zharkikh, E. V., Kozlov, I. O., Zherebtsov, E. A., Potapova, E. V., Malaya, N. S., Yakushkina, N. Y., Snimshchikova, I. A., and Dunaev, A. V., "Peculiarities of local blood microcirculation in patients with psoriasis," Proceedings of SPIE 10685, 1068532 (2018).

[9] Mizeva, I., Makovik, I., Dunaev, A., Krupatkin, A., and Meglinski, I., "Analysis of skin blood microflow oscillations in patients with rheumatic diseases," Journal of Biomedical Optics 22(7) (2017).

[10] Zherebtsov, E., Zherebtsova, A., Doronin, A., Dunaev, A., Podmasteryev, K., Bykov, A., and Meglinski, I., "Combined use of laser doppler flowmetry and skin thermometry for functional diagnostics of intradermal finger vessels," Journal of Biomedical Optics 22, 22 - 22 - 4 (2017).

[11] Zherebtsova, A. I., Zherebtsov, E. A., Dunaev, A. V., Podmasteryev, K. V., Pilipenko, O. V., Krupatkin, A. I., Khakhicheva, L. S., and Muradyan, V. F., "Study of the functional state of peripheral vessels in fingers of rheumatological patients by means of laser doppler flowmetry and cutaneous thermometry measurements," Proc.SPIE 9917, 9917 - 9917 - 7 (2016).

[12] Lancaster, G., Stefanovska, A., Pesce, M., Marco Vezzoni, G., Loggini, B., Pingitore, R., Ghiara, F., Barachini, P., Cervadoro, G., Romanelli, M., and Rossi, M., "Dynamic markers based on blood perfusion fluctuations for selecting skin melanocytic lesions for biopsy," Scientific Reports 5, 12825 (2015).

[13] Ogrin, R., Darzins, P., and Khalil, Z., "Age-related changes in microvascular blood flow and transcutaneous oxygen tension under basal and stimulated conditions," Journals of Gerontology - Series A Biological Sciences and Medical Sciences 60(2), 200-206 (2005).

[14] Tikhonova, I., Tankanag, A., and Chemeris, N., "Age-related changes of skin blood flow during postocclusive reactive hyperemia in human," Skin Research and Technology 19(1), e174-e181 (2013).

[15] Nagashima, Y., Yada, Y., Suzuki, T., and Sakai, A., "Evaluation of the use of an integration-type laserdoppler flowmeter with a temperature-loading instrument for measuring skin blood flow in elderly subjects during cooling load: comparison with younger subjects," International Journal of Biometeorology 47, 139147 (May 2003).

[16] Tikhonova, I. V., Tankanag, A. V., and Chemeris, N. K., "Timeamplitude analysis of skin blood flow oscillations during the post-occlusive reactive hyperemia in human," Microvascular Research 80(1), $58-64$ (2010).

[17] Zherebtsov, E., Sokolovski, S., Sidorov, V., Rafailov, I., Dunaev, A., and Rafailov, E. U., "Novel wearable VCSEL-based blood perfusion sensor," Proceedings - International Conference Laser Optics 2018, ICLO 8435409, 564 (2018).

[18] Urbancic-Rovan, V., Stefanovska, A., Bernjak, A., Azman-Juvan, K., and Kocijancic, A., "Skin blood flow in the upper and lower extremities of diabetic patients with and without autonomic neuropathy," Journal of Vascular Research 41(6), 535-545 (2004).

[19] Johnson, J. M. and Kellogg, D. L., "Local thermal control of the human cutaneous circulation," Journal of Applied Physiology 109(4), 1229-1238 (2010).

[20] Hodges, G. J., Mallette, M. M., Tew, G. A., Saxton, J. M., Moss, J., Ruddock, A. D., and Klonizakis, M., "Effect of age on cutaneous vasomotor responses during local skin heating," Microvascular Research 112, 47-52 (2017).

[21] Baboshina, N. V., "Parameters of microcirculation in both sexes at different ages," Human Physiology 44, 466-473 (Jul 2018). 